Pacific

Journal of

Mathematics

MULTI-VARIABLE POLYNOMIAL SOLUTIONS TO PELL'S EQUATION AND FUNDAMENTAL UNITS IN REAL QUADRATIC FIELDS

J. MC Laughlin 


\title{
MULTI-VARIABLE POLYNOMIAL SOLUTIONS TO PELL'S EQUATION AND FUNDAMENTAL UNITS IN REAL QUADRATIC FIELDS
}

\author{
J. MC LAUGHLiN
}

Solving Pell's equation is of relevance in finding fundamental units in real quadratic fields and for this reason polynomial solutions are of interest in that they can supply the fundamental units in infinite families of such fields.

In this paper an algorithm is described which allows one to construct, for each positive integer $n$, a finite collection, $\left\{F_{i}\right\}$, of multi-variable polynomials (with integral coefficients), each satisfying a multi-variable polynomial Pell's equation

$$
C_{i}^{2}-F_{i} H_{i}^{2}=(-1)^{n-1},
$$

where $C_{i}$ and $H_{i}$ are multi-variable polynomials with integral coefficients. Each positive integer whose square-root has a regular continued fraction expansion with period $n+1$ lies in the range of one of these polynomials. Moreover, the continued fraction expansion of these polynomials is given explicitly as is the fundamental solution to the above multi-variable polynomial Pell's equation.

Some implications for determining the fundamental unit in a wide class of real quadratic fields is considered.

\section{Introduction.}

Solving Pell's equation is of relevance in finding fundamental units in real quadratic fields and for this reason polynomial solutions are interesting in that they can supply the fundamental units in infinite families of such fields.

There have been several papers written over the past thirty years which describe certain polynomials whose square roots have periodic continued fraction expansions which can be written down explicitly in terms of the coefficients and variables of the polynomials. See for example the papers of Bernstein [1], Levesque and Rhin [4], Madden [5], Van der Poorten [10] and Van der Poorten and Williams [11].

In this paper an algorithm is described which allows one to construct, for each positive integer $n$, a finite collection of multi-variable FermatPell polynomials which have all positive integers whose square-roots have a continued fraction expansion of period $n+1$ in their range. If $F_{i}:=$ 
$F_{i}\left(t_{0}, t_{1}, \ldots, t_{\left\lfloor\frac{n+1}{2}\right\rfloor}\right)$ is any one of these polynomials, the fundamental polynomial solution to the equation

$$
C_{i}^{2}-F_{i} H_{i}^{2}=(-1)^{n-1}
$$

(where $C_{i}$ and $H_{i}$ are polynomials in the variables $t_{0}, t_{1}, \ldots, t_{\left\lfloor\frac{n+1}{2}\right\rfloor}$ ) can be found. Moreover, the continued fraction expansion of $\sqrt{F_{i}}$ can be written down when $t_{1}, \ldots, t_{\left\lfloor\frac{n+1}{2}\right\rfloor} \geq 0$ and $t_{0}>g_{i}\left(t_{1}, \ldots, t_{\left\lfloor\frac{n+1}{2}\right\rfloor}\right)$, a certain rational function of these variables. Some implications for single-variable FermatPell polynomials are discussed as are the implications for writing down the fundamental units in a wide class of real quadratic number fields.

Definition. A multi-variable polynomial

$$
F:=F\left(t_{0}, t_{1}, \ldots, t_{k}\right) \in \mathbb{Z}\left[t_{0}, t_{1}, \ldots, t_{k}\right], k \geq 1
$$

is called a multi-variable Fermat-Pell polynomial ${ }^{1}$ if there exists polynomials

$$
C:=C\left(t_{0}, t_{1}, \ldots, t_{k}\right) \text { and } H:=H\left(t_{0}, t_{1}, \ldots, t_{k}\right) \in \mathbb{Z}\left[t_{0}, t_{1}, \ldots, t_{k}\right]
$$

such that either

$$
\begin{aligned}
& C^{2}-F H^{2}=1, \text { for all } t_{i}, \quad 0 \leq i \leq k, \text { or } \\
& C^{2}-F H^{2}=-1, \text { for all } t_{i}, \quad 0 \leq i \leq k .
\end{aligned}
$$

Such a triple of polynomials $\{C, H, F\}$ satisfying Equation (1.2) constitute a multi-variable polynomial solution to Pell's equation.

Definition. The multi-variable Fermat-Pell polynomial $F$ (as above) is said to have a multi-variable polynomial continued fraction expansion if there exists a positive integer $n$, a real constant $T$, a rational function $g\left(t_{1}, \ldots, t_{k}\right) \in$ $\mathbb{Q}\left(t_{1}, \ldots, t_{k}\right)$ and polynomials $a_{0}:=a_{0}\left(t_{0}, t_{1}, \ldots, t_{k}\right) \in \mathbb{Z}\left[t_{0}, t_{1}, \ldots, t_{k}\right]$ and $a_{j}:=a_{j}\left(t_{1}, \ldots, t_{k}\right) \in \mathbb{Z}\left[t_{1}, \ldots, t_{k}\right], 1 \leq j \leq n$, which take only positive integral values for integral $t_{i} \geq T, 1 \leq i \leq k$ and (possibly half-) integral $t_{0}>g\left(t_{1}, \ldots, t_{k}\right)$ such that

$\sqrt{F}=\left[a_{0} ; \overline{a_{1}, \ldots, a_{n}, 2 a_{0}}\right]$, for all $t_{i}$ 's in the ranges stated, $0 \leq i \leq k$.

\section{Remarks.}

(1) From the point of view of simplicity it would be desirable to replace the condition $t_{0} \geq g\left(t_{1}, \ldots, t_{k}\right)$ by $t_{0} \geq T$ but it will be seen that for the polynomials examined here that the former condition is more natural and indeed cannot be replaced by the latter condition.

\footnotetext{
${ }^{1}$ These polynomials are called "Fermat-Pell polynomials" here to avoid confusion with "Pell Polynomials" and also because Fermat investigated the "Pell" equation.
} 
(2) The restriction that the $a_{i}\left(t_{1}, \ldots, t_{k}\right) \geq 0,1 \leq i \leq n$ may also seem artificial to some since negative terms can easily be removed from a continued fraction expansion (see, for example [10]) but this changes the period of the continued fraction so is avoided here.

(3) It may also seem artificial to have $a_{0}$ depend on a variable $t_{0}$ while the other $a_{i}$ 's do not but this will also be seen to occur naturally.

(4) Finally, allowing $t_{0}$ to take half-integral values in some circumstances may also seem strange but this also will be seen to be natural and indeed necessary.

Definition. If, for all sets of integers $\left\{t_{0}^{\prime}, t_{1}^{\prime}, \ldots, t_{k}^{\prime}\right\}$ satisfying $t_{0}^{\prime} \geq g\left(t_{1}^{\prime}\right.$, $\left.\ldots, t_{k}^{\prime}\right)$ and $t_{i}^{\prime} \geq T, 1 \leq i \leq k$,

$$
X=C_{i}\left(t_{0}^{\prime}, t_{1}^{\prime}, \ldots, t_{k}^{\prime}\right), \quad Y=H_{i}\left(t_{0}^{\prime}, t_{1}^{\prime}, \ldots, t_{k}^{\prime}\right)
$$

constitutes the fundamental solution (in integers) to

$$
X^{2}-F_{i}\left(t_{0}^{\prime}, t_{1}^{\prime}, \ldots, t_{k}^{\prime}\right) Y^{2}=(-1)^{n-1}
$$

then $\left(C_{i}\left(t_{0}, t_{1}, \ldots, t_{k}\right), H_{i}\left(t_{0}, t_{1}, \ldots, t_{k}\right)\right)$ is termed the fundamental polynomial solution to Equation (1.1).

Standard notations are used:

$$
a_{0}+\frac{1}{a_{1}+} \frac{1}{a_{2}+} \frac{1}{a_{3}+} \ldots \frac{1}{a_{N}}:=a_{0}+\frac{1}{a_{1}+\frac{1}{a_{2}+\frac{1}{a_{3}+\ldots \frac{1}{a_{N}}}} .} .
$$

To save space this continued fraction is usually written $\left[a_{0} ; a_{1}, \ldots, a_{n}\right]$. The infinite periodic continued fraction with initial non-periodic part $a_{0}$ and periodic part $a_{1}, \ldots, a_{n}, 2 a_{0}$ is denoted by $\left[a_{0} ; \overline{a_{1}, \ldots, a_{n}, 2 a_{0}}\right]$. The $i$-th approximant of the continued fraction $\left[a_{0} ; a_{1}, \ldots,\right]$ is denoted by $P_{i} / Q_{i}$.

Repeated use will be made of some basic facts about continued fractions, such as:

$$
\begin{aligned}
& P_{n} Q_{n-1}-P_{n-1} Q_{n}=(-1)^{n-1}, \\
& P_{n+1}=a_{n+1} P_{n}+P_{n-1}, \\
& Q_{n+1}=a_{n+1} Q_{n}+Q_{n-1},
\end{aligned}
$$

each of these relations being valid for $n=1,2,3 \ldots$

Before coming to the main problem, it is necessary to first solve a related problem on symmetric strings of positive integers. 


\section{A problem concerning symmetric sequences.}

Question: For which symmetric sequences of positive integers $a_{1}, \ldots, a_{n}$ do there exist positive integers $a_{0}$ and $D$ such that

$$
\sqrt{D}=\left[a_{0} ; \overline{a_{1}, \ldots, a_{n}, 2 a_{0}}\right] ?
$$

Let $P_{i} / Q_{i}$ denote the $i$ th approximant of the continued fraction

$$
0+\frac{1}{a_{1}+} \frac{1}{a_{2}+} \frac{1}{a_{3}+} \ldots \frac{1}{a_{n}} .
$$

By the well-known correspondence between convergents and matrices

$$
\begin{aligned}
\left(\begin{array}{ll}
0 & 1 \\
1 & 0
\end{array}\right)\left(\begin{array}{cc}
a_{1} & 1 \\
1 & 0
\end{array}\right) \ldots \ldots\left(\begin{array}{cc}
a_{n} & 1 \\
1 & 0
\end{array}\right) & =\left(\begin{array}{ll}
P_{n} & P_{n-1} \\
Q_{n} & Q_{n-1}
\end{array}\right) \\
\Longrightarrow\left(\begin{array}{ll}
0 & 1 \\
1 & 0
\end{array}\right)\left(\begin{array}{cc}
a_{1} & 1 \\
1 & 0
\end{array}\right) \ldots \ldots\left(\begin{array}{cc}
a_{n} & 1 \\
1 & 0
\end{array}\right)\left(\begin{array}{ll}
0 & 1 \\
1 & 0
\end{array}\right) & =\left(\begin{array}{ll}
P_{n-1} & P_{n} \\
Q_{n-1} & Q_{n}
\end{array}\right) .
\end{aligned}
$$

Since the left side in the second equation is a symmetric sequence of symmetric matrices it follows that

$$
P_{n}=Q_{n-1}
$$

Suppose $\sqrt{D}=\left[a_{0} ; \overline{a_{1}, \ldots \ldots, a_{n}, 2 a_{0}}\right]=a_{0}+\beta$, where $\beta=\left[0 ; \overline{a_{1}, \ldots \ldots, a_{n}, 2 a_{0}}\right]$ so that

$$
\begin{aligned}
& \beta=\left[0 ; a_{1}, \ldots, a_{n}, 2 a_{0}+\beta\right], \\
\Longrightarrow & \beta=\frac{\left(2 a_{0}+\beta\right) P_{n}+P_{n-1}}{\left(2 a_{0}+\beta\right) Q_{n}+Q_{n-1}}=\frac{\beta P_{n}+\left(2 a_{0} P_{n}+P_{n-1}\right)}{\beta Q_{n}+\left(2 a_{0} Q_{n}+Q_{n-1}\right)}, \\
\Longrightarrow & \beta^{2} Q_{n}+\left(2 a_{0} Q_{n}+Q_{n-1}-P_{n}\right) \beta-\left(2 a_{0} P_{n}+P_{n-1}\right)=0, \\
\Longrightarrow & \beta^{2} Q_{n}+\left(2 a_{0} Q_{n}\right) \beta-\left(2 a_{0} P_{n}+P_{n-1}\right)=0,(\text { by }(2.3)) \\
\Longrightarrow & \sqrt{D}=a_{0}+\beta=\sqrt{a_{0}^{2}+\frac{2 a_{0} P_{n}+P_{n-1}}{Q_{n}}} .
\end{aligned}
$$

The problem now becomes one of determining for which symmetric sequences of positive integers $a_{1}, \ldots, a_{n}$ does there exist positive integers $a_{0}$ such that $\left(2 a_{0} P_{n}+P_{n-1}\right) / Q_{n}$ is an integer.

Theorem 1. There exists a positive integer $a_{0}$ such that $\left(2 a_{0} P_{n}+P_{n-1}\right) / Q_{n}$ is an integer if and only if $P_{n-1} Q_{n-1}$ is even.

Proof. $\Longleftarrow$ Suppose first of all that $P_{n-1} Q_{n-1}$ is even. By Equation (1.3)

$$
P_{n} Q_{n-1}+(-1)^{n}=P_{n-1} Q_{n} .
$$


(i) Suppose $n$ is even. Then $P_{n} Q_{n-1} P_{n-1}+P_{n-1}=P_{n-1}^{2} Q_{n}$. Choose $t$ to be any integer or half-integer such that $t Q_{n}$ is an integer and $a_{0}:=$ $Q_{n-1} P_{n-1} / 2+t Q_{n}>0$. Then

$$
\frac{2 a_{0} P_{n}+P_{n-1}}{Q_{n}}=\frac{Q_{n-1} P_{n-1} P_{n}+2 t P_{n} Q_{n}+P_{n-1}}{Q_{n}}=2 t P_{n}+P_{n-1}^{2} .
$$

(ii) Similarly, in the case $n$ is odd, $-P_{n} Q_{n-1} P_{n-1}+P_{n-1}=-P_{n-1}^{2} Q_{n}$. Choose $t$ to be any integer or half-integer such that $t Q_{n}$ is an integer and $a_{0}:=-Q_{n-1} P_{n-1} / 2+t Q_{n}>0$. In this case

$$
\frac{2 a_{0} P_{n}+P_{n-1}}{Q_{n}}=2 t P_{n}-P_{n-1}^{2} .
$$

$\Longrightarrow$ Suppose next that $P_{n-1}$ and $Q_{n-1}$ are both odd and that there exists a positive integer $a_{0}$ such that $\left(2 a_{0} P_{n}+P_{n-1}\right) / Q_{n}$ is a positive integer, $m$, say. Using (1.3) and (2.3) it follows that $Q_{n}$ is even. Then $2 a_{0} P_{n}+P_{n-1}=m Q_{n}$ implies $P_{n-1}$ is even - a contradiction.

\section{Remarks.}

(i) Note that this process gives all $a_{0}$ such that $\left(2 a_{0} P_{n}+P_{n-1}\right) / Q_{n}$ is an integer. Indeed,

$$
\begin{aligned}
& \left(2 a_{0} P_{n}+P_{n-1}\right) / Q_{n}=k, \text { an integer } \\
& \Longleftrightarrow 2 a_{0} P_{n} Q_{n-1}=-P_{n-1} Q_{n-1}+k Q_{n} Q_{n-1}, \\
& \left.\Longleftrightarrow 2 a_{0}(-1)^{n-1}=2 a_{0}\left(P_{n} Q_{n-1}-P_{n-1} Q_{n}\right), \quad \text { (by }(1.3)\right) \\
& =-P_{n-1} Q_{n-1}+Q_{n}\left(k Q_{n-1}-2 a_{0} P_{n-1}\right), \\
& \Longleftrightarrow a_{0}=(-1)^{n-1}\left(\frac{-P_{n-1} Q_{n-1}}{2}+Q_{n} \frac{k Q_{n-1}-2 a_{0} P_{n-1}}{2}\right) .
\end{aligned}
$$

Notice also that if there is one such $a_{0}$ that there are infinitely many of them.

(ii) Notice that, with $P_{n}, P_{n-1}, Q_{n}$ and $Q_{n-1}$ as defined above, if there exists a positive integer $D$ satisfying (2.1) then $D=p\left(t_{0}\right)$, for some allowed $t_{0}$, where

$$
\begin{aligned}
& p(t)=\left(\frac{Q_{n-1} P_{n-1}}{2}+t Q_{n}\right)^{2}+2 t P_{n}+P_{n-1}^{2}, t>\frac{-Q_{n-1} P_{n-1}}{2 Q_{n}}, \quad(n \text { even }), \\
& p(t)=\left(\frac{-Q_{n-1} P_{n-1}}{2}+t Q_{n}\right)^{2}+2 t P_{n}-P_{n-1}^{2}, t>\frac{Q_{n-1} P_{n-1}}{2 Q_{n}}, \quad(n \text { odd }) .
\end{aligned}
$$

The above theorem suggests a simple algorithm for deciding if, for a given symmetric sequence of positive integers $a_{1}, \ldots, a_{n}$, there exist positive integers $a_{0}$ and $D$ such that (2.1) holds. Notice that all that matters is the parity of the $a_{i}$ so all calculations can be done in $\mathbb{Z}_{2}$. First of all define the 
following matrices:

$$
J=\left(\begin{array}{ll}
0 & 1 \\
1 & 0
\end{array}\right), K=\left(\begin{array}{ll}
1 & 1 \\
1 & 0
\end{array}\right) \text { and } I=\left(\begin{array}{ll}
1 & 0 \\
0 & 1
\end{array}\right) .
$$

Convert the sequence $a_{1}, a_{2}, \ldots, a_{n}$ to a sequence of $J$ - and $K$-matrices, according to whether each $a_{i}$ is odd (replace by a $K$ ) or even (replace by a $J$ ). Prefix a $J$-matrix (to account for the initial 0 in the continued fraction (2.2)). Multiply this sequence together (modulo 2) using the facts that $J^{2}=K^{3}=$ $I$, and $J K=K^{2} J$.

The final matrix $\equiv\left(\begin{array}{ll}* & 1 \\ * & 1\end{array}\right) \bmod 2 \Longleftrightarrow$ there do not exist positive integers $a_{0}$ and $D$ such that $(2.1)$ holds.

Example 1. Do there exist positive integers $a_{0}$ and $D$ such that

$$
\sqrt{D}=\left[a_{0} ; \overline{22,34,97,32,15,17,17,15,32,97,34,22,2 a_{0}}\right] ?
$$

As described above convert the sequence $22,34,97,32,15,17,17,15,32,97$, 34,22 to a sequence of $J$ - and $K$-matrices, prefix a $J$-matrix and multiply the sequence together:

$$
\begin{aligned}
\underbrace{J J} J K J \underbrace{K K K} K J K \underbrace{J J}=J K(J K) J K & =J \underbrace{K\left(K^{2}\right.} \underbrace{J) J} K \\
& =J K=\left(\begin{array}{ll}
1 & 0 \\
1 & 1
\end{array}\right) .
\end{aligned}
$$

Therefore there do exist positive integers $a_{0}$ and $D$ such that

$$
\sqrt{D}=\left[a_{0} ; \overline{22,34,97,32,15,17,17,15,32,97,34,22,2 a_{0}}\right] .
$$

\section{Multi-variable Fermat-Pell polynomials.}

Definition. If $\left\{a_{1}, \ldots, a_{n}\right\}$ is a symmetric zero-one sequence such that

$$
\left(\begin{array}{ll}
0 & 1 \\
1 & 0
\end{array}\right) \prod_{i=1}^{n}\left(\begin{array}{cc}
a_{i} & 1 \\
1 & 0
\end{array}\right) \not \equiv\left(\begin{array}{cc}
* & 1 \\
* & 1
\end{array}\right) \quad \bmod 2
$$

then the sequence $\left\{a_{1}, \ldots, a_{n}\right\}$ is termed a permissible sequence. Let $r(n)$ denote the number of permissible sequences of length $n$.

Note: It is not difficult to show that $r(2 m)=\left((-1)^{m}+2^{m+1}\right) / 3$ and that $r(2 m+1)=\left((-1)^{m}+5 \times 2^{m}\right) / 3$.

If $D$ is a positive integer such that $\sqrt{D}=\left[a_{0} ; \overline{a_{1}, \ldots, a_{n}, 2 a_{0}}\right]$ then $\left\{a_{1}\right.$, $\left.\ldots, a_{n}\right\} \bmod 2 \operatorname{must}$ equal one of the above permissible sequences and $D$ is said to be associated with this permissible sequence. The collection of all positive integers associated with a particular permissible sequence is termed the parity class of this permissible sequence. Sometimes, if there is no danger of ambiguity, these collections of positive integers will be referred to simply as parity classes. 


\section{Theorem 2.}

(i) For each positive integer $n$ there exists a finite collection of multivariable Fermat-Pell polynomials $F_{j}\left(t_{0}, t_{1}, \ldots, t_{\left\lfloor\frac{n+1}{2}\right\rfloor}\right), 1 \leq j \leq r(n)$, such that each positive integer whose square root has a continued fraction expansion with period $n+1$ lies in the range of exactly one of these polynomials. Moreover, these polynomials can be constructed.

(ii) These polynomials have a polynomial continued fraction expansion which can be explicitly determined.

(iii) The fundamental polynomial solution

$$
\begin{gathered}
C=C_{j}\left(t_{0}, t_{1}, \ldots, t_{\left\lfloor\frac{n+1}{2}\right\rfloor}\right), H=H_{j}\left(t_{0}, t_{1}, \ldots, t_{\left\lfloor\frac{n+1}{2}\right\rfloor}\right) \text { to } \\
C^{2}-F_{j}\left(t_{0}, t_{1}, \ldots, t_{\left\lfloor\frac{n+1}{2}\right\rfloor}\right) H^{2}=(-1)^{n-1}
\end{gathered}
$$

exists and can be explicitly determined.

Proof. (i) The proof will be by construction.

Step 1. Find all permissible sequences. This will involve checking $2^{\left\lfloor\frac{n+1}{2}\right\rfloor}$ zero-one sequences in a way similar to Example 1 above.

Step 2. For each permissible sequence $\left\{a_{1}, \ldots, a_{n}\right\}$ create a new symmetric polynomial sequence $\left\{a_{1}\left(t_{1}\right), a_{2}\left(t_{2}\right), \ldots, a_{n-1}\left(t_{2}\right), a_{n}\left(t_{1}\right)\right\}$ by replacing each $a_{i}$ and its partner $a_{n+1-i}$ in the symmetric sequence by $a_{i}\left(t_{i}\right)=a_{n+1-i}\left(t_{i}\right)=$ $2 t_{i}+1$ if $a_{i}=1$ and by $a_{i}\left(t_{i}\right)=a_{n+1-i}\left(t_{i}\right)=2 t_{i}+2$ if $a_{i}=0$. This new sequence will sometimes be referred to as the sequence $\left\{a_{1}, \ldots, a_{n}\right\}$, if there is no danger of ambiguity. Each of the integer variables $t_{i}$ (in the polynomial being constructed) will be allowed to vary independently over the range $0 \leq t_{i}<\infty$ and each of the new $a_{i}$ 's will keep the same parity and stay positive.

Step 3. As in (2.2), form the continued fraction

$$
0+\frac{1}{a_{1}\left(t_{1}\right)+} \frac{1}{a_{2}\left(t_{2}\right)+} \cdots \frac{1}{a_{n-1}\left(t_{2}\right)+} \frac{1}{a_{n}\left(t_{1}\right)}
$$

and calculate $P_{n}, Q_{n}, P_{n-1}$ and $Q_{n-1}$ for this polynomial continued fraction, where these expressions are now polynomials in the $t_{i}$ 's.

Step 4. Construct $F_{j}:=F_{j}\left(t_{0}, t_{1}, \ldots, t_{\left\lfloor\frac{n+1}{2}\right\rfloor}\right)$, the multi-variable FermatPell polynomial corresponding to the particular parity sequence under consideration. This is simply done by defining

$$
F_{j}:= \begin{cases}\left(\frac{Q_{n-1} P_{n-1}}{2}+t_{0} Q_{n}\right)^{2}+2 t_{0} P_{n}+P_{n-1}^{2}, & (n \text { even }) \\ \left(\frac{-Q_{n-1} P_{n-1}}{2}+t_{0} Q_{n}\right)^{2}+2 t_{0} P_{n}-P_{n-1}^{2}, & (n \text { odd })\end{cases}
$$

where $(-1)^{n+1} Q_{n-1} P_{n-1} /\left(2 Q_{n}\right)<t_{0}<\infty$ and $t_{0}$ can take half-integral values if $Q_{n}$ is even and otherwise takes integral values. 
Every positive integer whose square root has a continued fraction expansion with period $n+1$ lies in the range of exactly one of these polynomials. That these polynomials are multi-variable Fermat-Pell polynomials follows from Equation (3.4) below.

(ii) With $t_{0}$ in the range given, then

$$
\sqrt{F_{j}}=\left[a_{0}\left(t_{0}, t_{1}, \ldots, t_{\left\lfloor\frac{n+1}{2}\right\rfloor}\right) ; \overline{a_{1}\left(t_{1}\right), \ldots, a_{n}\left(t_{1}\right), 2 a_{0}\left(t_{0}, t_{1}, \ldots, t_{\left\lfloor\frac{n+1}{2}\right\rfloor}\right)}\right],
$$

for all $t_{i} \geq 0$. Here

$$
a_{0}=a_{0}\left(t_{0}, t_{1}, \ldots, t_{\left\lfloor\frac{n+1}{2}\right\rfloor}\right):= \begin{cases}\frac{Q_{n-1} P_{n-1}}{2}+t_{0} Q_{n}, & (n \text { even }) \\ \frac{-Q_{n-1} P_{n-1}}{2}+t_{0} Q_{n}, & (n \text { odd }) .\end{cases}
$$

(iii) Notice (using (1.3) and (2.3)) that

$$
\left(a_{0} Q_{n}+P_{n}\right)^{2}-\left(a_{0}^{2}+\left(2 a_{0} P_{n}+P_{n-1}\right) / Q_{n}\right) Q_{n}^{2}=(-1)^{n-1} .
$$

To see that $\left(a_{0} Q_{n}+P_{n}, Q_{n}\right)$ is the fundamental solution to (3.1), notice that

$$
\sqrt{F_{j}}=\left[a_{0}\left(t_{0}, t_{1}, \ldots, t_{\left\lfloor\frac{n+1}{2}\right\rfloor}\right) ; \overline{a_{1}\left(t_{1}\right), \ldots, a_{n}\left(t_{1}\right), 2 a_{0}\left(t_{0}, t_{1}, \ldots, t_{\left\lfloor\frac{n+1}{2}\right\rfloor}\right)}\right] .
$$

This has period $n+1$ and the $n$th approximant is $a_{0}+P_{n} / Q_{n}=\left(a_{0} Q_{n}+\right.$ $\left.P_{n}\right) / Q_{n}$ and by the theory of the Pell equation $\left(a_{0} Q_{n}+P_{n}, Q_{n}\right)$ is the fundamental solution to (3.1).

As regards fundamental units in quadratic fields there is the following theorem on page 119 of [6]:

Theorem 3. Let $D$ be a square-free, positive rational integer and let $K=$ $\mathbb{Q}(\sqrt{D})$. Denote by $\epsilon_{0}$ the fundamental unit of $K$ which exceeds unity, by $s$ the period of the continued fraction expansion for $\sqrt{D}$, and by $P / Q$ the $(s-1)$-th approximant of it.

If $D \not \equiv 1 \bmod 4$ or $D \equiv 1 \bmod 8$, then

$$
\epsilon_{0}=P+Q \sqrt{D} \text {. }
$$

However, if $D \equiv 5 \bmod 8$, then

$$
\epsilon_{0}=P+Q \sqrt{D}
$$

or

$$
\epsilon_{0}^{3}=P+Q \sqrt{D}
$$

Finally, the norm of $\epsilon_{0}$ is positive if the period $s$ is even and negative otherwise. 
It is easy, working modulo 4 , to determine simple conditions (on $t_{0}$ ) which make $F_{j} \equiv 2$ or $3 \bmod 4$ and thus to say further, for a particular set of choices of $t_{1}, \ldots, t_{\left\lfloor\frac{n+1}{2}\right\rfloor}$ and for all odd or even $t_{0}$, that if $F_{j}$ is square-free, then $a_{0} Q_{n}+P_{n}+\sqrt{F_{j}} Q_{n}$ is the fundamental unit in $\mathbb{Q}\left[\sqrt{F_{j}}\right]$. For example, suppose that $n$ is even and that the original $Q_{n-1}$ determined from the permissible zero-one sequence is also even (so that $P_{n-1}$ and $Q_{n}$ are both odd and $P_{n}=Q_{n-1}$ is even). Then the multi-variable form of $Q_{n-1}$ evaluated in Step 3 will also have all even coefficients. Suppose $\frac{Q_{n-1}}{2} \equiv c_{0}+\sum t_{i^{\prime}} \bmod 2$. (Here $c_{0}$ may be 0 and the sum $\sum t_{i^{\prime}}$ may contain some, all or none of the $t_{i}$ 's.) It is easy to see that $F_{j}\left(t_{0}, t_{1}, \ldots, t_{\left\lfloor\frac{n+1}{2}\right\rfloor}\right) \equiv\left(c_{0}+\sum t_{i^{\prime}}+t_{0}\right)^{2}+1$ mod 4. Even more simply, if the original $Q_{n-1}$ as in Step 1 is odd (here also the case $n$ is even is considered) then $P_{n-1}$ as evaluated in Step 3 is even and it is not difficult to show that in fact $P_{n-1} \equiv 2 \bmod 4$ (since for $n$ even $P_{n} Q_{n-1}-P_{n-1} Q_{n}=-1$ ) and that $Q_{n}$ is odd, which leads to $F_{j}\left(t_{0}, t_{1}, \ldots, t_{\left\lfloor\frac{n+1}{2}\right\rfloor}\right) \equiv t_{0}^{2}+1 \bmod 4$. Similar relations hold in the case where $n$ is odd.

The polynomials constructed in Theorem 2 take values in only one parity class, if all the variables are positive. However, given any two parity classes, there are multi-variable Fermat-Pell polynomials that take values in those two classes.

Theorem 4. Let $n$ be any fixed positive integer large enough so that the set of positive integers whose square roots have a continued fraction expansion of period $n+1$ can be divided into more than one parity class.

(i) Given any two parity classes of integers whose square roots have continued fraction expansions of period $n+1$, there are multi-variable Fermat-Pell polynomials, which can be constructed, that take values in both parity classes.

(ii) These polynomials have a polynomial continued fraction expansion which can be explicitly determined.

(iii) If $F=F\left(t_{0}, c, t_{1}, \ldots, t_{\left\lfloor\frac{n+1}{2}\right\rfloor}\right)$ is any such polynomial then the fundamental polynomial solution

$$
C=C\left(t_{0}, c, t_{1}, \ldots, t_{\left\lfloor\frac{n+1}{2}\right\rfloor}\right), H=H\left(t_{0}, c, t_{1}, \ldots, t_{\left\lfloor\frac{n+1}{2}\right\rfloor}\right)
$$

to

$$
C^{2}-F H^{2}=(-1)^{n-1}
$$

can be explicitly determined.

Proof. As in Step 2 in Theorem 2 a polynomial sequence $\left\{a_{1}, \ldots, a_{n}\right\}$ is created. Suppose $L_{1}=\left\{b_{1} \ldots, b_{n}\right\}$ and $L_{2}=\left\{c_{1}, \ldots, c_{n}\right\}$ are the permissible sequences associated with the two parity classes. Let $i_{1}, \ldots, i_{k}$ be 
those positions $\leq\left\lfloor\frac{n+1}{2}\right\rfloor$ at which the sequences agree. For each of these $i_{r}$ 's set $a_{i_{r}}\left(t_{i_{r}}\right)=a_{n+1-i_{r}}\left(t_{i_{r}}\right)=2 t_{i_{r}}+1$, if $c_{i_{r}}$ is odd and set $a_{i_{r}}\left(t_{i_{r}}\right)=$ $a_{n+1-i_{r}}\left(t_{i_{r}}\right)=2 t_{i_{r}}+2$, if $c_{i_{r}}$ is even. Subdivide the remaining positions (those positions $\leq\left\lfloor\frac{n+1}{2}\right\rfloor$ at which $L_{1}$ and $L_{2}$ differ) into two subsets: Those at which $L_{1}$ has a 0 and $L_{2}$ has a 1 and those at which $L_{1}$ has a 1 and $L_{2}$ has a 0 .

Suppose $i_{j}$ is a position of the first kind. Let $a_{i_{j}}\left(c, t_{i_{j}}\right)=a_{n+1-i_{j}}\left(c, t_{i_{j}}\right)$ $=c+2+2 t_{i_{j}}$. Repeat this for all the positions $i_{j}$ in this first set. Likewise, suppose $i_{j}$ is a position of the second kind. In this case let $a_{i_{j}}\left(c, t_{i_{j}}\right)=$ $a_{n+1-i_{j}}\left(c, t_{\left\lfloor\frac{n+1}{2}\right\rfloor}\right)=c+1+2 t_{i_{j}}$. This is also repeated for all the positions $i_{j}$ in this second set. Step 3 and Step 4 are then carried out as above. The rest of the proof is identical to Theorem 2. Denote the polynomial produced by

$$
F:=F\left(t_{0}, c, t_{1}, \ldots, t_{\left\lfloor\frac{n+1}{2}\right\rfloor}\right) .
$$

As in Theorem 2, if $c$ and all the $t_{i}$ 's are nonnegative, $1 \leq i \leq\left\lfloor\frac{n+1}{2}\right\rfloor$ and $t_{0}>(-1)^{n+1} Q_{n-1} P_{n-1} /\left(2 Q_{n}\right)$ then

$$
\sqrt{F}=\left[a_{0} ; \overline{a_{1}, \ldots \ldots, a_{n}, 2 a_{0}}\right],
$$

where the $a_{i}$ 's, $1 \leq i \leq n$ are as defined just above and $a_{0}$ is as defined in Equation (3.3).

Under these conditions also the parity class of $F\left(t_{0}, c, t_{1}, \ldots, t_{\left\lfloor\frac{n+1}{2}\right\rfloor}\right)$ will depend only on the parity of $c$. As in Theorem 2 the fundamental polynomial solution to

$$
C^{2}-F\left(t_{0}, c, t_{1}, \ldots, t_{\left\lfloor\frac{n+1}{2}\right\rfloor}\right) H^{2}=(-1)^{n-1}
$$

is given by $C=a_{0} Q_{n}+P_{n}, H=Q_{n}$.

\section{A worked example.}

As an example, consider those positive integers whose square-roots have continued fraction expansion with period of length 9 . Thus the symmetric part of the period has length 8 and it is necessary to check the $2^{4}=16$ zero-one sequences to determine which are permissible. (This checking is done in essentially the same way as in Example 1 above.) There are 11 valid sequences:

$$
\begin{aligned}
& 0,0,0,0,0,0,0,0 \\
& 0,0,0,1,1,0,0,0 \\
& 0,0,1,1,1,1,0,0 \\
& 0,1,0,0,0,0,1,0
\end{aligned}
$$




$$
\begin{aligned}
& 0,1,0,1,1,0,1,0 \\
& 0,1,1,1,1,1,1,0 \\
& 1,0,0,1,1,0,0,1 \\
& 1,0,1,0,0,1,0,1 \\
& 1,0,1,1,1,1,0,1 \\
& 1,1,0,0,0,0,1,1 \\
& 1,1,1,0,0,1,1,1 .
\end{aligned}
$$

The ninth of these is considered in more detail (each of the others can be dealt with in a similar way). For clarity the letters $a, b, c$ and $d$ are used instead of $t_{1}, t_{2}, t_{3}$ and $t_{4}$. Evaluating the continued fraction

$$
0+\frac{1}{2 a+1+\frac{1}{2 b+2+\frac{1}{2 d+1+\frac{1}{2 d+1+\frac{1}{2 c+1+\frac{1}{2 b+2+\frac{1}{2 a+1}}}}}}}
$$

it is found that

$$
\begin{aligned}
& P_{8}=Q_{7}=-1-2 d+ \\
& 2(3+4 a+2 b+4 a b)(4+3 b+4 c+4 b c+6 d+4 b d+8 c d+8 b c d) \\
& +4(3+2 b+4(1+b) c)(2+b+3 c+2 b c+a(3+2 b+4(1+b) c)) \times \\
& \left(1+2 d+2 d^{2}\right), \\
& P_{7}=4(1+b)(4+3 b+4 c+4 b c+6 d+4 b d+8 c d+8 b c d)+ \\
& 2(3+2 b+4(1+b) c)^{2}\left(1+2 d+2 d^{2}\right) \text { and } \\
& Q_{8}=8(2+b+3 c+2 b c+a(3+2 b+4(1+b) c))^{2}\left(1+2 d+2 d^{2}\right)+ \\
& (3+4 a+2 b+4 a b)(3+4 c+4 d+8 c d+(2+4 a)(4+3 b+ \\
& 4 c+4 b c+6 d+4 b d+8 c d+8 b c d)) .
\end{aligned}
$$

Since $n$ is 8 (even) and $Q_{8}$ is odd (so $t_{0}$ cannot take half-integer values), in this case $F_{9}\left(t_{0}, a, b, c, d\right)$ is defined by

$$
F_{9}\left(t_{0}, a, b, c, d\right)=\left(Q_{7} P_{7} / 2+t_{0} Q_{8}\right)^{2}+2 t_{0} P_{8}+P_{7}^{2}
$$


and

$$
\sqrt{F_{9}\left(t_{0}, a, b, c, d\right)}=\left[Q_{7} P_{7} / 2+\frac{t_{0} Q_{8} ; \overline{2 a+1,2 b+2,2 c+1,2 d+1,2 d+1},}{2 c+1,2 b+2,2 a+1,2\left(Q_{7} P_{7} / 2+t_{0} Q_{8}\right)}\right],
$$

this expansion being valid for all $a, b, c, d \geq 0$ and all $t_{0}>-Q_{7} P_{7} /\left(2 Q_{8}\right)$ and in particular for all $t_{0} \geq 0$. In these ranges

$$
C=\left(Q_{7} P_{7} / 2+t_{0} Q_{8}\right) Q_{8}+P_{8}, H=Q_{8}
$$

gives the fundamental polynomial solution to

$$
C^{2}-F_{9} H^{2}=-1 \text {. }
$$

$F_{9}\left(t_{0}, a, b, c, d\right)=\left(Q_{7} P_{7} / 2+t_{0} Q_{8}\right)^{2}+2 t_{0} P_{8}+P_{7}^{2} \equiv\left(1+t_{0}^{2}\right) \bmod 4$, so that if $\left(Q_{7} P_{7} / 2+t_{0} Q_{8}\right)^{2}+2 t_{0} P_{8}+P_{7}^{2}$ is a square-free number for some particular $a, b, c, d \geq 0$ and some odd $t_{0}>-Q_{7} P_{7} /\left(2 Q_{8}\right)$, then

$$
\left(Q_{7} P_{7} / 2+t_{0} Q_{8}\right) Q_{8}+P_{8}+\sqrt{\left(Q_{7} P_{7} / 2+t_{0} Q_{8}\right)^{2}+2 t_{0} P_{8}+P_{7}^{2}} Q_{8}
$$

is the fundamental unit in $\mathbb{Q}\left(\sqrt{\left(Q_{7} P_{7} / 2+t_{0} Q_{8}\right)^{2}+2 t_{0} P_{8}+P_{7}^{2}}\right)$.

\section{Mystification, Fermat-Pell polynomials of a single variable and more on odd-even.}

Clearly it is possible to "mystify" this process by replacing each $t_{i}$ by some polynomial $g_{i}\left(t_{i}\right)$ taking only positive values or by replacing $2 t_{i}$ (recalling that the continued fraction expansion contains only terms like $2 t_{i}+1$ or $\left.2 t_{i}+2\right)$ by some polynomial $g_{i}\left(t_{i}\right)$ taking only even nonnegative values or by setting $t_{i}=t_{i}\left(X_{1}, X_{2}, \ldots, X_{k}\right), 1 \leq i \leq\left\lfloor\frac{n+1}{2}\right\rfloor$, a polynomial in the $X_{j}$ 's taking only positive values, where the $X_{j}$ 's can be independent variables and $k$ can be as large as desired and so on.

Finally of course one can obtain single-variable Fermat-Pell polynomials by replacing the original variables $t_{0}, t_{i}, 1 \leq i \leq\left\lfloor\frac{n+1}{2}\right\rfloor$ by polynomials in a single variable. If it is desired that the period of the continued fraction expansion of the new single-variable Fermat-Pell polynomial should stay the same as that of the originating multi-variable polynomial then the domain of the single variable should be restricted so that the polynomials replacing each of the $t_{i}$ 's take only positive values as in the multi-variable case and the polynomial replacing $t_{0}$ must be such that the $a_{0}$ term stays positive for all allowed values of the new single variable.

For example, letting $a=s, b=0, c=s, d=0$ and $t_{0}=s$ in the polynomial (4.2) above produces the single-variable Fermat-Pell polynomial

$$
\begin{aligned}
& g(s)=639557+6858268 s+33078145 s^{2}+ \\
& 94534688 s^{3}+177380352 s^{4}+228442240 s^{5}+204593408 s^{6}+
\end{aligned}
$$




$$
125870080 s^{7}+50925568 s^{8}+12238848 s^{9}+1327104 s^{10}
$$

which has the continued fraction expansion (valid for all $s \geq 0$ )

$$
\begin{aligned}
\sqrt{g(s)} & =\left[799+4289 s+9184 s^{2}+9856 s^{3}+5312 s^{4}+1152 s^{5}\right. \\
& \left.\frac{\overline{2 s+1,2,2 s+1,1,1,2 s+1,2,2 s+1,}}{2\left(799+4289 s+9184 s^{2}+9856 s^{3}+5312 s^{4}+1152 s^{5}\right)}\right] .
\end{aligned}
$$

$g(s) \equiv\left(1+s^{2}\right) \bmod 4$ so when $s$ is odd and positive and $g(s)$ is square-free

$$
\begin{aligned}
& 51982+534625 s+2429840 s^{2}+6408000 s^{3}+ \\
& 10812928 s^{4}+12115200 s^{5}+9019392 s^{6}+4304896 s^{7}+1196032 s^{8}+ \\
& 147456 s^{9}+\sqrt{g(s)}\left(65+320 s+576 s^{2}+448 s^{3}+128 s^{4}\right)
\end{aligned}
$$

is the fundamental unit in $\mathbb{Q}[\sqrt{g(s)}]$. For example, letting $s=1$ gives that $47020351+1537 \sqrt{935888258}$ is the fundamental unit in $\mathbb{Q}[\sqrt{935888258}]$.

Starting with the continued fraction

$$
0+\frac{1}{2 a+1+\frac{1}{2 b+2+\frac{1}{2 d+1+\frac{1}{2 d+1+\frac{1}{c+2 e+\frac{1}{2 a+1}}}}}}
$$

and following the same steps as above with the continued fraction (4.1) a multi-variable Fermat-Pell polynomial is developed which takes values in the parity classes associated with permissible sequences 7 and 9 . Letting $a=b=d=e=t=0$ one gets the single-variable Fermat-Pell polynomial

$$
\begin{gathered}
g(c)=4325+28140 c+83652 c^{2}+147440 c^{3}+168000 c^{4}+ \\
126528 c^{5}+61504 c^{6}+17664 c^{7}+2304 c^{8}
\end{gathered}
$$

with continued fraction expansion

$$
\sqrt{g(c)}=\left[65+\frac{214 c+288 c^{2}+184 c^{3}+48 c^{4} ;}{1,2, c, 1,1, c, 2,1,2\left(65+214 c+288 c^{2}+184 c^{3}+48 c^{4}\right)}\right],
$$

valid for $c \geq 1$. 


\section{Concluding remarks.}

Every Fermat-Pell polynomial in one variable, $s$ say, that eventually has a continued fraction expansion of fixed period length can be found from (3.2), if it takes values in only one parity class for all sufficiently large $s$, and from (3.6), if it takes values in two parity class for all sufficiently large $s$. (Recall Remark (i) after Theorem 1.)

Of course none of this does anything to answer Schinzel's question of whether every Fermat-Pell polynomial in one variable has a continued fraction expansion. Neither does it provide a criterion (such as Schinzel's in the degree-two case) for deciding if a polynomial of arbitrarily high even degree is a Fermat-Pell polynomial. Perhaps it raises another question - Does every multi-variable Fermat-Pell polynomial have a continued fraction expansion? Does every multi-variable Fermat-Pell polynomial have a continued fraction expansion, assuming every Fermat-Pell polynomial in one variable does?

\section{References}

[1] L. Bernstein, Fundamental units and cycles in the period of real quadratic number fields, I, Pacific J. Math., 63(1) (1976), 37-61, MR 53 \#13166, Zbl 0335.10010.

[2] _ Fundamental units and cycles in the period of real quadratic number fields, II, Pacific J. Math., 63(1) (1976), 63-78, MR 53 \#13167, Zbl 0335.10011.

[3] L. Euler (Translated by John D. Blanton), Introduction to Analysis of the Infinite Book I, Springer-Verlag, New York, Berlin, Heidelberg, London, Tokyo, 1988 (Orig. 1748), MR g:01067, Zbl 0657.01013.

[4] C. Levesque and G. Rhin, A few classes of periodic continued fractions, Utilitas Math., 30 (1986), 79-107, MR 88b:11072, Zbl 0615.10014.

[5] D.J. Madden, Constructing families of long continued fractions, Pacific J. Math, 198(1) (2001), 123-147, MR 2002b:11014.

[6] W. Narkiewicz, Elementary and Analytic Theory of Algebraic Numbers (Second Edition), Springer-Verlag, New York, Berlin, Heidelberg, London, Tokyo, Hong Kong/PWN-Polish Scientific Publishers, Warszawa, 1990 (First Edition 1974), MR 91h:11107, Zbl 0717.11045.

[7] O. Perron, Die Lehre von dem Kettenbrüchen, B.G. Teubner, Leipzig-Berlin, 1913.

[8] A. Schinzel, On some problems of the arithmetical theory of continued fractions, Acta Arith., 6 (1960/1961), 393-413, MR 23 \#A3111, Zbl 0099.04003.

[9] _ On some problems of the arithmetical theory of continued fractions, II, Acta Arith., 7 (1961/1962), 287-298, MR 25 \#2998.

[10] A.J. van der Poorten, Explicit formulas for units in certain quadratic number fields, Algorithmic number theory (Ithaca, NY, 1994), 194-208, Lecture Notes in Comput. Sci., 877, Springer, Berlin, 1994, MR 96b:11146, Zbl 0837.11004.

[11] A.J. van der Poorten and H.C. Williams, On certain continued fraction expansions of fixed period length, Acta Arith., 89(1) (1999), 23-35, MR 2000m:11010, Zbl 0926.11005. 
Received April 13, 2001 and revised July 16, 2002.

Mathematics Department

Trinity College

300 Summit Street

HARTFORD, CT 06106-3100

E-mail address: james.mclaughlin@trincoll.edu 